Eine empirische Analyse am Beispiel von BUND-Mitgliedern

\title{
Was Umweltbewusstsein (nicht) bewirken kann
}

\section{Die Bedeutung des organisierten Umweltschutzes für die Umweltpolitik ist unbestritten: Umweltverbände haben erheblich zur breiten Politisierung der Ökologiediskussion beigetragen. Welchen Einfluss sie auf Konsumprozesse haben, ist dagegen weit weniger bekannt. Wie verhalten sich die Mitglieder eines Umweltschutzverbandes in ihrer Rolle als Konsumenten? Können sie als ökologieorientierte Konsuminnovatoren wirken?}

$\mathrm{D}$ Von Achim Spiller er Markt für umwelffreundliche Erzeugnisse stagniert seit einigen Jahren (bei Öko-Textilien) oder wächst nur verhalten (bei Lebensmitteln). In einer Studie wurde der Frage nachgegangen, welchen Einfluss organisierte Umweltschützer auf die weitere Marktentwicklung nehmen können (1). Die Befragung von Umweltverbandsmitgliedern ist insofern spannend, als es sich hier um Personen handelt, an deren ökologischer Einstellung wenig Zweifel besteht. Messprobleme des Umweltbewusstseins, die ansonsten die Forschung dominieren, können deshalb in den Hintergrund rücken. Der Schwerpunkt der Untersuchung lag auf der Handlungsebene. Es ist plausibel anzunehmen, dass Umweltverbandsmitglieder die Grenzen dessen vorgeben, was derzeit innerhalb der Gesellschaft im Rahmen des privaten Handelns erwartetwerden kann (2). Diejenigen Verhaltensweisen, die nicht einmal von langjährigen Umweltverbandsmitgliedern ausgeübt werden, dürften in absehbarer Zeit schwerlich in der allgemeinen Bevölkerung Fuß fassen.

Durch die Zusammenarbeit mit dem Bund für Umwelt und Naturschutz Deutschland e. V. (BUND) bestand ein direkter Kontakt zu den Mitgliedern eines der führenden Umweltschutzverbände. Die schriftliche Befragung wurde 1997 durchgeführt. Vergleichswerte zur Einordnung dieser Ergebnisse lieferte eine im Vorfeld (1996) durchgeführte allgemeine (mündliche) Bevölkerungsbefragung (3).

\section{Hohes Umweltbewusstsein}

Innerhalb der allgemeinen Bevölkerung wurde insgesamt ein sehr niedriger Umweltwissensstand festgestellt (4). Im Vergleich dazu sind die Kenntnisse der BUND-Mitglieder wesentlich detaillierter tierten Handelsbetrieben und Textilanbietern aus. Die relativ hohen Werte alternativer Anbieter bei der Befragung sind ein aussagekräftiger Indikator für umweltorientierte Einkaufsbemühungen und -erfahrungen.

Auch die emotionale Bewertung der Umweltproblematik unterscheidet sich zwischen beiden Gruppen deutlich. Erwartungsgemäß ist das Umweltbewusstsein der BUND-Mitglieder - hier spezifiziert als emotionale Betroffenheit und Umweltwissen - ausgeprägt. Zu fragen ist, inwieweit es innerhalb der Strukturvorgaben der modernen Konsumgesellschaft handlungsrelevant wird.

\section{Umwelthandeln beim Konsum}

und zwar auch dann, wenn man berücksichtigt, dass der Wissensstand bei einer schriftlichen Befragung durch Bedenkzeit und Informationsmöglichkeiten generell höher ausgewiesen wird. So geben bei der Frage nach Kennzeichen für die Umweltfreundlichkeit von Lebensmitteln nur Wenige den Grünen Punkt an (3,4 Prozent gegenüber 24,4 Prozent in der Gesamtbevölkerung). Deutlich häufiger werden dagegen die Kennzeichen des ökologischen Landbaus erwähnt (Demeter: 52,9 Prozent gegenüber 9,8 Prozent, Bioland 43,7 Prozent gegenüber 9,4 Prozent). Ähnlich fällt das Ergebnis bei den Fragen nach umweltorien-
Der Schwerpunkt der Studie lag auf der Analyse des privaten Umwelthandelns. Abbildung 1 skizziert einen Teil der Ergebnisse und deutet zunächst auf die überraschende Erkenntnis hin, dass es zwar einen Unterschied im Niveau der Umweltschutzbemühungen, aber nicht in der Struktur gibt. Offensichtlich haben Umweltverbandsmitglieder und Gesamtbevölkerung in den gleichen Handlungsfeldern Schwierigkeiten, ihr Konsumverhalten zu ändern. Bereiche wie Mülltrennung und Verpackung stehen allseits im Vordergrund.

\section{Abb. 1: Vergleichendes Unweliprofil von BUND-Mitgliedern und Gesamfbevölkerung}

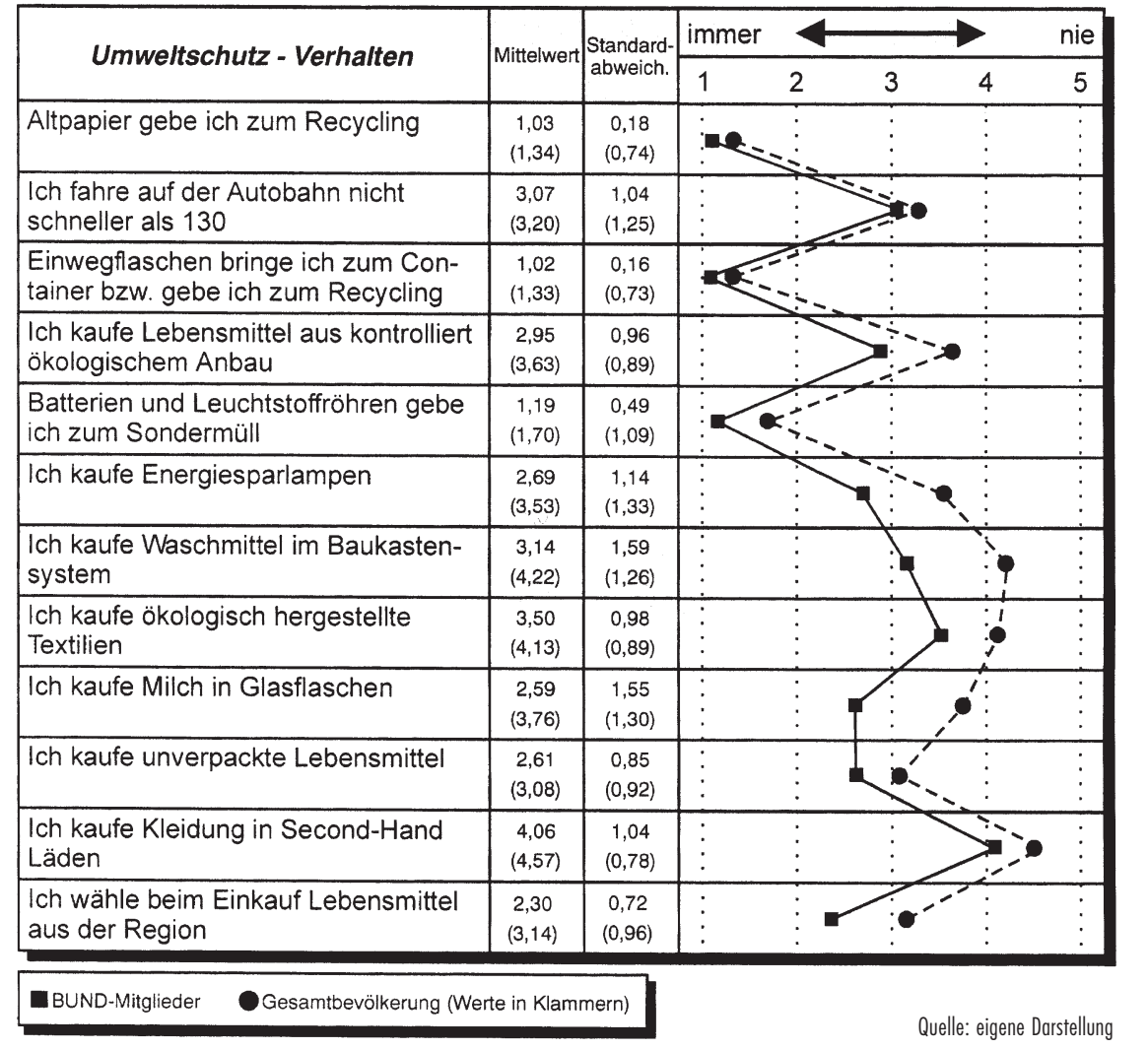


Dieses Grundmuster - Unterschiede im Niveau, aber nicht in der Struktur - bleibt prinzipiell auch dann erhalten, wenn man die zentralen Konsumfelder Mobilität, Wohnen, Ernährung und Bekleidung vertieft analysiert; es zeigen sich allerdings einige aufschlussreiche Details.

\section{- Beispiele: Mobilität}

Vielfältige Studien referieren den außerordentlich geringen Einfluss des Umweltbewusstseins auf das Verkehrsverhalten. So resümiert Franzen (5): Auf die tägliche Verkehrsmittelwahl zum Arbeitsort, die Höhe der gefahrenen Jahreskilometer und den Benzinkonsum übt das Umweltbewusstsein keinen, auf die Auto-Anschaffungsentscheidung, die Nutzungshäufigkeit pro Woche, die Wahl des Verkehrsmittels für den Haushaltseinkauf und das Abschalten des Motors bei Wartezeiten einen schwachen Einfluss aus. Diese negativen Resultate werden bei der Befragung von BUND-Mitgliedern nur zum Teil bestätigt, insgesamt ist eine Verkehrswende bei der Untersuchungsgruppe aber nicht zu erkennen:

- Die Anzahl der Autos pro Haushalt liegt etwas unter dem Bevölkerungsdurchschnitt; diese Abweichung ist aber nicht signifikant.

- Die Fahrleistung liegt bei 11.700 Kilometern im Jahr gegenüber 14.308 Kilometern in der Gesamtbevölkerung und ist damit signifikant geringer.

Positiv ist die signifikant stärkere Nutzung der Bahn.

- Das Flugzeug wird vor allem europa- und weltweit weniger genutzt; es gibt weniger Vielflieger.

Gleichzeitig zeigen aber zwei andere Fragen, dass die BUND-Mitglieder sehr wohl um die ökologische Relevanz des Verkehrs wissen. Bei der offenen - Frage nach den Einschränkungen, die sie zugunsten des Umweltschutzes in Kauf nehmen, wird in erster Linie der Mobilitätsbereich genannt (55 Prozent der Nennungen). Auch bei der Frage nach den persönliche Handlungsbarrieren steht das Verkehrsverhalten an erster Stelle (47 Prozent der Nennungen). Es wird deutlich, dass die Form der Mobilität als zentrales Umweltschutzproblem in dieser Zielgruppe erkannt ist. Man weiß um die Problematik speziell der automobilen Fortbewegung und des Flugverkehrs, ist zugleich aber mit seinem eigenen Verhalten unzufrieden. Die Dissonanz zwischen erkannter Problemrelevanz auf der einen und dem eigenen Handlungsbeitrag auf der anderen Seite ist ausgeprägt und wird von den
Umweltverbandsmitgliedern auch als solche wahrgenommen. Dies ist ein deutlicher Hinweis auf die vielfältigen praktischen Widerstände, die von den einzelnen Haushalten nur ansatzweise überwunden werden können. Individuelle Verhaltensänderungen stoßen hier an ihre Grenzen.

\section{- Wohnverhalten}

Ähnlich fallen die Ergebnisse im Wohnbereich aus. Eine Vergleichbarkeit zum Bevölkerungsdurchschnitt ist hier aufgrund des hohen Anteils der ländlich lebenden BUND-Mitglieder nur bedingt gegeben. Dennoch deutet sich an, dass es hinsichtlich der Wohnform (Haustyp), der Wohnfläche pro Kopf und der genutzten Energieträger keine positiven Abweichungen gibt. Beim Stromverbrauch liegen die Umweltverbandsmitglieder dagegen signifikant günstiger; hier machen sich möglicherweise Einsparbemühungen bezahlt.

\section{Ernährung}

Im Gegensatz zum Verkehrs- und Wohnbereich sind beim Ernährungsverhalten relevante Unterschiede zur Gesamtbevölkerung zu erkennen. So liegt der energieintensive Fleischkonsum signifikant niedriger (18 Prozent Vegetarier gegenüber 4,2 Prozent in der Gesamtstichprobe); auch Tiefkühlprodukte werden seltener genutzt. Besonders aufschlussreich ist das Kaufverhalten bei Lebensmitteln aus kontrolliert ökologischem Anbau. 70,5 Prozent der Befragten geben an, solche Erzeugnisse relativ häufig zu kaufen. Der Unterschied zum Durchschnitt der Bevölkerung ist hoch signifikant und dürfte in der Realität noch größer sein, weil im Gegensatz zur Gesamtbevölkerung eine deutliche Korrelation zwischen der Kenntnis umweltbezogener Lebensmittelkennzeichen und dem angegebenen Kaufverhalten vorhanden ist, mithin weniger der Effekt, dass die Antwort durch soziale Erwünschtheit beeinflusst wird, vorliegt.

\section{Textilien}

Bei einem in der Selbsteinschätzung niedrigeren Modebewusstsein ist der Verbrauch an Kleidung bei den BUND-Mitgliedern geringer als in der Gesamtbevölkerung. Sie greifen zudem etwas häufiger zu Second-Hand-Kleidung, wenn auch insgesamt auf niedrigem Niveau. Signifikante Unterschiede zeigen sich insbesondere beim Kauf ökologischer Textilien: Zumindest manchmal kaufen 48,4 Prozent der BUND-Mitglieder solche Produkte (26,6 Prozent gesamt). Dies korrespondiert mit dem größeren Wissen um entspre- chende Öko-Label. Insgesamt sind die Unterschiede im Textilbereich ausgeprägt. Während in der Gesamtbevölkerung kaum Ansätze zum umweltfreundlichen Verhalten zu erkennen sind und der Marktanteil ökologischer Textilien entsprechend niedrig liegt, haben BUND-Mitglieder die Bedeutung dieses Themenfeldes offensichtlich wahrgenommen. Hierauf deutet der hohe Bekanntheitsgrad entsprechender Anbieter hin.

\section{Differenzierung nach Bereichen nötig}

Ein pauschales Urteil zum ökologieorientierten Lebensstil der Umweltverbandsmitglieder und damit zum Zusammenhang zwischen Umweltbewusstsein und -verhalten lässt sich nicht fällen. Vielmehr zeigt sich eine große Diskrepanz zwischen den einzelnen Handlungsfeldern. Das Bemühen der BUND-Mitglieder um individuellen Umweltschutz ist unverkennbar und schlägt sich besonders im Abfall-, Ernährungs- und Textilbereich nieder. Hier erweist sich das erheblich ausgeprägtere Umweltwissen als handlungsrelevant. Vergleicht man den Kauf ökologischer Produkte in den beiden Gruppen, so kann man durchaus Ansätze einer Konsumwende erkennen.

Anders sieht der Zusammenhang für Mobilitätsund Wohnentscheidungen aus. Die wenigen Fortschritte schlagen sich in der Gesamtbelastung kaum nieder. Angesichts der hohen umweltpolitischen Bedeutung gerade dieser beiden Handlungsfelder ist das Resultat ernüchternd. Die Gründe lassen sich nur vermuten:

- Die offenen Fragen nach Lebensstileinschränkungen und Handlungsbarrieren zeigen, dass die Bedeutung des Verkehrsbereichs den BUND-Mitgliedern in hohem Maße bewusst ist. Auffällig ist, dass auch die ökologische Belastung des Flugverkehrs von den BUND-Mitgliedern wahrgenommen wird. Immerhin 20 Prozent der Befragten geben dieses Feld bei den offenen Fragen an. Auch die Verhaltensalternativen dürften hinlänglich bekannt sein. Die Reichweite individueller Bemühungen - die Kunst des richtigen Verhaltens in den falschen Strukturen - scheint hier an ihre Grenzen zu stoßen (6). Ökonomisch-strukturelle Veränderungen wie die Verbreitung des Ferntourismus sind stärker als individuelle Handlungsbemühungen.

- Auch BUND-Mitgliedern dürfte die ökologische Bedeutung des Wohnens nur ansatzweise, nämlich in Bezug auf den Energieverbrauch, deutlich sein. Wohnungsform und -größe wer- 
den bei den offenen Fragen nach Einschränkungs- und Barrierefeldern nur ein einziges Mal genannt, obwohl sie wesentliche Folgen für die Ökobilanz des Haushalts haben. Ähnliche Ergebnisse bei der Befragung der Gesamtbevölkerung deuten darauf hin, dass hinsichtlich der ökologischen Relevanz der Wohnstruktur eine gesellschaftliche Wissenslücke vorliegt und erheblicher Aufklärungsbedarf besteht.

- Die hohe Übereinstimmung in der Struktur des umweltrelevanten Konsums bekräftigt die Ergebnisse der Lebensstil- und Milieuforschung, dass ein alternatives Milieu im Sinne der 70er und 80er Jahre kaum mehr aufzufinden ist (7). Organisierte Umweltschützer konsumieren nicht grundsätzlich anders, sondern leisten in Form einer „Stïckwerktechnologie“ partielle Beiträge im Rahmen der gesellschaftlich üblichen Lebensstile. Eine Avantgarde, die innerhalb der bestehenden Strukturen weitreichende Beispiele nachhaltigen Konsums vorlebt, sind sie im Allgemeinen nicht.

\section{- Hartnäckige Hemmnisse}

Es ist problematisch, dass mit den beiden Bereichen Wohnen und Mobilität zwei aus gesamtökologischer Sicht besonders prioritäre Felder sich dem privaten Umwelthandeln hartnäckig widersetzen. Das Verhalten ist hier nicht so sehr durch das Umweltbewusstsein, sondern durch Hintergrundvariablen wie das Einkommen geprägt. Wohnen und Verkehr sind in vielfältiger Form aneinander gekoppelt und zeichnen sich dadurch aus, dass die entsprechenden Konsumentscheidungen in aller Regel langfristiger Natur sind. Es handelt sich um strategische Konsumentscheidungen, die den grundsätzlichen Weg vorgeben, auf dem sich die tägliche Konsumpraxis bewegt. Sie gehen zumeist auf einen extensiven Entscheidungsprozess zurück, sind auf Kontinuität angelegt und kurzfristig aufgrund der hohen spezifischen Investitionen kaum zu variieren. Hausbau, Wohnortwahl, Hobbys, Heizungstechnologie und ähnliche Entscheidungen sind auch angesichts neuer ökologischer Anforderungen kaum noch zu ändern. Investitionen, die sich nur mit Wertverlust revidieren lassen, begründen Abhängigkeit. Solche sogenannten sunk costs sind die zentrale ökonomische Ursache für Beharrungseffekte (8).

Neben Sachkapitalinvestitionen sind es im Konsumbereich insbesondere Humankapitalinvestitionen, von denen Bindungseffekte ausgehen. Am
Beispiel der Wohnortwahl lässt sich dies verdeutlichen: Bei einem ökologisch sinnvollen Wohnungswechsel (beispielsweise um näher an den Arbeitsplatz zu gelangen) gehen möglicherweise Freundschaften verloren, die über Jahre hinweg gepflegt wurden. Ein wichtiger Grund für die steigende Wohnungsgröße pro Kopf ist der Verbleib in der alten Wohnung, auch dann, wenn sich wesentliche Lebensumstände (etwa: Auszug der Kinder, Scheidung) und damit der Bedarf geändert haben. Die Bindung an das soziale Umfeld, Vertrautheit und der Gewöhnungseffekt lassen die eigentlich zu groß gewordene Wohnung weiterhin attraktiv erscheinen.

\section{Politische Schlussfolgerungen}

Für die ökologische Konsumforschung ist zu fordern, dass sie hinsichtlich operativer und strategischer Konsumentscheidungen unterschiedliche politische Handlungsstrategien entwickelt. In den Feldern Abfall, Ernährung und Textilien deutet das Verhalten der BUND-Mitglieder darauf hin, dass bei einem guten Informationsstand und einem hinreichenden, preiswerten Angebot wichtige Beiträge aus der Konsumsphäre kommen können. Staatliche Hilfestellungen, unternehmerische Initiativen und privater Umweltschutz können hier - trotz aller Widerstände - langfristig ein Netzwerk sich selbst tragender Verbesserungen bilden. Nach der „Foot in the door-Technik“ sollte im Rahmen eines Sozialmarketings die schrittweise Verbreitung der entsprechenden Handlungsweisen propagiert werden (9). Organisierte Umweltschützer sind hier wichtige Innovatoren. Sie in ihren Bemühungen zu unterstützen und ihr demonstratives Konsumverhalten in einem zur Zeit wenig ökologiefreundlichen Umfeld zum Beispiel mittels Anreizen und Vernetzungen gezielt zu fördern, ist ein bisher vernachlässigtes Feld staatlicher Umweltpolitik.

Bei strategischen Konsumentscheidungen wie Wohnform oder Mobilitätsstruktur muss dagegen die Führungsrolle eindeutig bei der Politik liegen. Die gesellschaftliche Einbindung sowie die Langfristigkeit und die Spezifität der Investitionen verlangen einen dominanten Akteur, der Handlungsanreize schafft und Trittbrettfahrerverhalten ausschließt. Angesichts der manifesten ökonomischen Interessen im Bereich Verkehr und der Zersplitterung des Wohnungsmarktes kann dies nur der Staat sein.

\section{Anmerkungen}

(1) Die Studie ist ausführlich dokumentiert in Bodenstein, G./ Elbers, H./ Spiller, A./ Zühlsdorf, A.: Umweltschützer als Zielgruppe des ökologischen Innovationsmarketing Ergebnisse einer Befragung von BUND-Mitgliedern, Diskussionsbeitrag des Fachbereichs Wirtschaftswissenschaft der Universität Duisburg Nr. 246, Duisburg 1998.

(2) Vgl. zu entsprechenden amerikanischen Studien de Haan, G./ Kuckartz, U.: Umweltbewusstsein, Opladen 1996, S. $105 \mathrm{ff}$.

(3) Auf Basis eines ausführlichen Fragebogens wurden Einstellungen und Verhaltensweisen für zahlreiche ökologische Handlungsfelder erhoben. Angeschrieben wurden nach einer Zufallsauswahl 250 Mitglieder des BUND, die über 18 Jahre alt sind und dem Verband mindestens fünf Jahre angehören. Die Rücklaufquote betrug 48,8 Prozent. Vgl. die ausführliche Dokumentation in Bodenstein, G./ Spiller, A./ Elbers, H.: Strategische Konsumentscheidungen: Langfristige Weichenstellungen für das Umwelthandeln - Ergebnisse einer empirischen Studie, Diskussionsbeitrag des Fachbereichs Wirtschaftswissenschaft der Universität Duisburg Nr. 234, Duisburg 1997.

(4) Vgl. auch Spiller, A.: Umweltbezogenes Wissen der Verbraucher: Ergebnisse einer empirischen Studie und Schlussfolgerungen für das Marketing, Diskussionsbeiträge des Fachbereichs Wirtschaftswissenschaft der Universitüt Duisburg Nr. 264, Duisburg 1999.

(5) Vgl. Franzen, A.: Umweltbewusstsein und Verkehrsverhalten, Zürich 1997, S. 141 1f.

(6) Vgl. Winterfeld, U. v.: Ökologisches Handeln - oder: Über die Kunst des richtigen Verhaltens in den falschen Strukturen, in: IÖW/VÖW Informationsdienst, Nr. 1/1992, S. 15.

(7) Vgl. die Milieustudien des Sinus-Instituts und Reusswig, F.: Die Gesellschaft der Lebensstile. Zur modernen Lebensstilforschung und ihrer ökologischen Bedeutung, in: Politische Ökologie, Nr. 33/ 1993, S. 6-9.

(8) Vgl. Picot, A./ Dietl, H./ Franck, E.: Organisation: Eine ökonomische Perspektive, Stuttgart 1997, S. 69 ff. (9) Vgl. auch Fejer, S./ Stroschein, F.-R.: Die Ableitung einer Guttman-Skala für sozial- und ökologiebewusstes Verhalten, in: planung und analyse, Nr. 1/1991, S. 5-12.

\section{Der Autor}

Dr. Achim Spiller ist Habilitand im Studiengang Marketing und Konsum der Gerhard Mercator Universität Duisburg.

Kontakt: Lotharstr. 65, 47057 Duisburg,

Tel. 0203/379-1234, Fax -2195,

E-mail: a.spiller@uni-duisburg.de 
(c) 20I0 Authors; licensee IÖW and oekom verlag. This is an article distributed under the terms of the Creative Commons Attribution Non-Commercial No Derivates License (http://creativecommons.org/licenses/by-nc-nd/3.o/), which permits unrestricted use, distribution, and reproduction in any medium, provided the original work is properly cited. 